\title{
Propagation and extinction of premixed edge-flames
}

\author{
David B. Clayton ${ }^{1}$, Min Suk Cha ${ }^{2}$ and Paul D. Ronney ${ }^{1 *}$ \\ ${ }^{1}$ Department of Aerospace and Mechanical Engineering \\ University of Southern California, Los Angeles, CA 90089-1453 \\ ${ }^{2}$ King Abdullah University of Science and Technology \\ Clean Combustion Research Center, Physical Science and Engineering Division \\ Thuwal 23955-6900, Saudi Arabia
}

\begin{abstract}
The propagation rates $\left(U_{\text {edge }}\right)$ of edge-flames in premixed hydrocarbon-oxygen-inert mixtures were measured as a function of global strain rate $(\sigma)$, mixture strength, and (by changing fuel and inert type) Lewis number $(\mathrm{Le})$. Using a counterflow slot-jet burner with electrical heaters at both ends to anchor the flame edges, both advancing (positive $U_{\text {edge}}$ ) and retreating (negative $U_{\text {edge}}$ ) edge-flames were characterized. Results are presented for both twin (premixed gas against premixed gas) and single (premixed gas against cold inert gas) edge-flames in terms of the effects of a non-dimensional strain rate $(\varepsilon)$ and non-dimensional heat loss $(\kappa)$ on a scaled propagation rate. $U_{\text {edge }}$ showed a strong dependence on $L e$ and flames images show that high (low) Le lead to weaker (stronger) edge-flame burning intensity. $U_{\text {edge }}$ for single edge-flames scaled with the square root of the unburned to burned gas density ratio in a manner similar to nonpremixed flames whereas for premixed flames $U_{\text {edge }}$ scaled linearly with density ratio. Edge-flames exhibited two extinction limits corresponding to a high- $\sigma$ strain induced limit and a low- $\sigma$ heat loss induced limit; a simple description of the low- $\sigma$ limits was proposed and found to correlate well with experiments in twin-premixed, single-premixed, and nonpremixed edge-flames over more than a two-decade range of $\kappa$. Results are in good qualitative and reasonable quantitative agreement with simple theories except that retreating twin edge-flames in high-Le mixtures are predicted theoretically but were not observed experimentally.
\end{abstract}

Keywords: edge-flames; premixed flames; extinction; Lewis number

*Corresponding author: ronney@usc.edu 


\section{Introduction}

In strong turbulence, flames may locally extinguish in areas where instantaneous strain rates are sufficiently large, causing edges separating burning and non-burning regions [1]. Depending on composition, temperature and flow properties these "edge-flames" [2, 3] may retreat further into burning regions (extinction fronts with negative edge-flame propagation speeds $\left(U_{\text {edge }}<0\right)$ ) or advance into unburned regions and restore flame surface (ignition fronts, $U_{\text {edge }}>0$ ). Moreover, edge-flames are an important aspect of premixed flame stabilization in shear layers [4]. Additionally, edge-flame libraries could potentially extend flamelet models of turbulent combustion, which assume unbroken flame surfaces, to conditions involving local quenching [5].

Perhaps the most canonical, readily-characterized configuration for studying edge-flames is plane strain with edges propagating in the third (unstrained) dimension. This configuration is employed in many theoretical and computational studies $[6,7,8]$ and is readily approximated experimentally using counterflowing slot-jets having large length-to-width ratio. This apparatus has been employed previously to study propagation rates and extinction limits of nonpremixed edge-flames $[9,10]$ where fuel flows from one jet and oxidant from the other. For premixed edge-flames two configurations are possible: premixed reactants flowing against premixed reactants, yielding twin premixed flames, and premixed reactants against inert gas, yielding single premixed flames. Theoretical studies of premixed edge-flames $[7,8]$ predict that $U_{\text {edge }}$ and extinction limits depend primarily on Lewis number $(L e)$ and two other dimensionless parameters representing strain and heat loss effects, respectively: $\varepsilon \equiv\left(\sigma \alpha / 2 S_{L}^{2}\right)^{1 / 2}$ and $\kappa \equiv \beta \alpha \kappa_{o} / S_{L}^{2}$, where $\sigma$ is the global strain rate, $\beta$ the non-dimensional activation energy (Zeldovich number), $\alpha$ the thermal diffusivity, $S_{L}$ the adiabatic unstrained laminar burning velocity and $\kappa_{o}$ the volumetric heat loss coefficient, estimated [9] for slot-jet counterflows as 7.5 $\alpha / d^{2}$, where $d$ is the slot-jet spacing. $\varepsilon^{2}$ is essentially a scaled Karlovitz number (ratio of strain rate to chemical reaction rate) and $\kappa^{1 / 2}$ a scaled Peclet number $\left(\kappa^{-1}\right.$ being a ratio of heat generation to heat loss). These theories predict 
premixed edge-flames exhibit two extinction limits corresponding to high- $\varepsilon$ strain-induced limits and low- $\varepsilon$ heat loss induced limits, with $U_{\text {edge }}<0$ for $\varepsilon$ close to these limits and $U_{\text {edge }}>0$ away from limits. At intermediate $\varepsilon$, theories predict $U_{\text {edge }}$ is nearly independent of $\varepsilon$, with $U_{\text {edge }} / S_{L} \approx 1$ for $L e=1$ and larger/smaller $U_{\text {edge }} / S_{L}$ for smaller/larger Le. These theories assume constant gas density; when thermal expansion effects are incorporated, for nonpremixed edge-flames theory predicts $U_{\text {edge }} / S_{L}$ (in the unburned gas reference frame far upstream) is accelerated in proportion to $\left(\rho_{u} / \rho_{b}\right)^{1 / 2}$ [11], where $\rho_{u}$ and $\rho_{b}$ are the unburned and burned gas densities, respectively. While this acceleration has been confirmed experimentally for nonpremixed flames $[9,10]$, it has not been determined whether $\left(\rho_{u} / \rho_{b}\right)^{1 / 2}$ scaling applies to premixed edge-flames.

While stationary $\left(U_{\text {edge }}=0\right)$ premixed edge-flames have been characterized experimentally [12, $13,14]$, no systematic studies of positive and negative speeds have been reported. Consequently, this work examines propagation rates and extinction limits of single and twin premixed edge-flames in slotjet counterflows for varying $\varepsilon, \kappa$ and $L e$. Both configurations are relevant to turbulent flames; single edge-flames are most relevant at lower turbulence intensities $\left(u^{\prime}\right)$ since wrinkled flame sheets have fresh reactants on one side and burned products on the other side, whereas twin edge-flames are relevant at higher $u^{\prime}$ where highly folded sheets with back-to-back flames may exist locally.

\section{Experimental apparatus}

The counterflow slot-jet apparatus and procedures are similar to those employed previously [9, 10]. Figure S1 shows a schematic of this apparatus. Thermal mass flow controllers regulated jet exit velocities $\left(U_{1}, U_{2}\right)$ to obtain specified mixtures and global strain rates $\sigma=\left(U_{1} / d\right)\left[1+\left(U_{2} / U_{1}\right)\left(\rho_{2} / \rho_{l}\right)^{1 / 2}\right]$, where $\left(\rho_{1}, \rho_{2}\right)$ are the gas densities at the jet exits [15]. Only $U$ (not $\rho$ ) was changed to vary $\sigma$ because changing $\rho$ would require changing the mixture which would substantially affect the other properties $(\alpha$, $S_{L}$, etc.) The jet velocities $U_{l}$ and $U_{2}$ were balanced; some spot-checks were performed with unequal $U_{l}$ 
and $U_{2}$ (but same $U_{1}+U_{2}$ ) and no significant differences were found except when $U_{1}$ or $U_{2}$ was so low that one of the flames became anchored to the jet exit, conditions outside regimes of interest for this investigation. Honeycomb inserts at the exits provide uniform flow across the jets' width (5 mm) and length $(130 \mathrm{~mm})$. Nitrogen sheath coflows with the same velocities were employed on both sides of both jets. The apparatus was maintained at room temperature with water cooling.

For conditions where $U_{\text {edge }}>0$, a nitrogen jet was used to extinguish or "erase" an established flame starting at one end, then the jet was retracted enabling the edge-flame to advance. For conditions causing $U_{\text {edge }}<0$, a mixture having $U_{\text {edge }}>0$ was introduced, then electrically-heated wires at both slot ends were activated, then mixture strength was slowly reduced to the desired value. The heated wires provided localized flame temperature enhancement, thus locally increasing reaction rates and anchoring the flame ends under conditions where they would retreat without heating. To induce extinction, the nitrogen jet was used to separate one flame end from its anchoring hot-wire, causing a retreating edgeflame. High-speed videos were analyzed to infer $U_{\text {edge, }}$ while an intensified CCD camera captured instantaneous images. The slot-jet aspect ratio is finite, consequently slight extensional flow occurs along the slot length which slightly affects $U_{\text {edge }}$ in the laboratory frame; this bias is nullified by interpolating least-square linear fits of $U_{\text {edge }}$ vs. position to the jet centerline $[9,10]$. At least three $U_{\text {edge }}$ measurements were taken at each condition; these were consistent within $\pm 5 \%$.

Lean $\mathrm{CH}_{4}$-air, lean $\mathrm{C}_{3} \mathrm{H}_{8}$-air and stoichiometric $\mathrm{CH}_{4}-\mathrm{O}_{2}$ mixtures diluted with $\mathrm{CO}_{2}$ having molar ratios 1:2:Q (Q denoting the dilution level) were employed to obtain compositions of various $S_{L}$ and effective $L e$ (ratio of mixture thermal diffusivity to mass diffusivity of the stoichiometrically-deficient reactant). Table 1 shows properties of these mixtures. $S_{L}$ was computed using PREMIX with USC Mech II chemical kinetics data [16]. The Zeldovich number $\beta$ is defined as $E\left(T_{a d}-T_{\infty}\right) /\left(R T_{a d^{2}}\right)$, where $T_{a d}$ is the adiabatic flame temperature, $T_{\infty}$ ambient temperature and $E / R$ activation temperature calculated from plots (not shown) of $\ln \left(S_{L}\right)$ vs. $1 / T_{a d}$, whose local slope is $-E / 2 R$. 
Table 1. Experimental conditions and corresponding flame properties for premixed edge-flames mixtures. Asterisks correspond to mixtures used to obtain the images shown in Figs. 1 and 4.

\begin{tabular}{|c|c|c|c|c|c|c|c|c|}
\hline \multicolumn{2}{|c|}{$\begin{array}{l}\text { Composition and } \\
\text { configuration }\end{array}$} & $\begin{array}{c}\text { Fuel } \% \text { or } \\
\text { Inert Ratio }(Q)\end{array}$ & $\begin{array}{c}S_{L} \\
(\mathrm{~cm} / \mathrm{s})\end{array}$ & $\begin{array}{c}d \\
(\mathrm{~cm})\end{array}$ & $\left(\rho_{u} / \rho_{b}\right)$ & $\begin{array}{c}\alpha \\
\left(\mathrm{cm}^{2} / \mathrm{s}\right)\end{array}$ & $\beta$ & $\kappa$ \\
\hline \multirow{11}{*}{$\begin{array}{l}\mathrm{Le}_{\text {fuel }}=0.96 \\
\mathrm{Le}_{\mathrm{O} 2}=1.10\end{array}$} & \multirow{6}{*}{ Twin } & 5.2 & 6.28 & \multirow{6}{*}{0.75} & 5.07 & 0.200 & 13.0 & 0.176 \\
\hline & & 5.3 & 6.94 & & 5.13 & 0.200 & 12.6 & 0.140 \\
\hline & & 5.6 & 9.18 & & 5.34 & 0.200 & 12.0 & 0.0763 \\
\hline & & $5.8 *$ & 10.8 & & 5.47 & 0.200 & 11.5 & 0.0531 \\
\hline & & 6.0 & 12.4 & & 5.60 & 0.200 & 10.9 & 0.0381 \\
\hline & & 6.5 & 16.8 & & 5.93 & 0.200 & 10.1 & 0.0191 \\
\hline & \multirow{5}{*}{ Single } & 6.9 & 20.8 & \multirow{5}{*}{0.75} & 2.48 & 0.200 & 9.64 & 0.0119 \\
\hline & & 7.2 & 23.8 & & 2.51 & 0.200 & 9.34 & 0.00888 \\
\hline & & 7.75 & 29.2 & & 2.58 & 0.200 & 9.08 & 0.00571 \\
\hline & & $8.3^{*}$ & 33.8 & & 2.64 & 0.200 & 9.06 & 0.00424 \\
\hline & & 9.0 & 38.8 & & 2.70 & 0.200 & 9.59 & 0.00342 \\
\hline \multirow{3}{*}{$\mathrm{C}_{3} \mathrm{H}_{8} /$ Air } & \multirow{3}{*}{ Twin } & 2.6 & 14.9 & \multirow{3}{*}{0.70} & 6.05 & 0.189 & 10.1 & 0.0251 \\
\hline & & 2.7 & 16.9 & & 6.21 & 0.189 & 9.69 & 0.0187 \\
\hline & & $2.8^{*}$ & 19.0 & & 6.37 & 0.188 & 9.36 & 0.0141 \\
\hline \multirow{3}{*}{$\begin{array}{l}\mathrm{Le}_{\text {fuel }}=1.86 \\
\mathrm{Le}_{\mathrm{O} 2}=1.05\end{array}$} & \multirow{3}{*}{ Single } & $3.2 *$ & 27.5 & \multirow{3}{*}{0.70} & 2.64 & 0.187 & 8.92 & 0.00637 \\
\hline & & 3.35 & 30.4 & & 2.68 & 0.186 & 8.81 & 0.00508 \\
\hline & & 3.55 & 34.0 & & 2.73 & 0.185 & 8.90 & 0.00406 \\
\hline \multirow{3}{*}{$\begin{array}{c}\mathrm{CH}_{4} / \mathrm{O}_{2} / \mathrm{CO}_{2} \\
=1 / 2 / Q\end{array}$} & \multirow{3}{*}{ Twin } & 7.15 & 4.45 & \multirow{3}{*}{0.50} & 6.12 & 0.121 & 14.5 & 0.319 \\
\hline & & 6.81 & 5.46 & & 6.25 & 0.122 & 14.2 & 0.213 \\
\hline & & $6.54 *$ & 6.40 & & 6.37 & 0.123 & 14.0 & 0.155 \\
\hline \multirow{3}{*}{$\begin{array}{l}\mathrm{Le}_{\text {fuel }}=0.74 \\
\mathrm{Le}_{\mathrm{O} 2}=0.86\end{array}$} & \multirow{3}{*}{ Single } & 5.96 & 8.85 & \multirow{3}{*}{0.50} & 2.58 & 0.125 & 13.5 & 0.0814 \\
\hline & & 5.57 & 11.0 & & 2.61 & 0.127 & 13.2 & 0.0532 \\
\hline & & $5.24 *$ & 13.1 & & 2.65 & 0.128 & 13.0 & 0.0375 \\
\hline
\end{tabular}




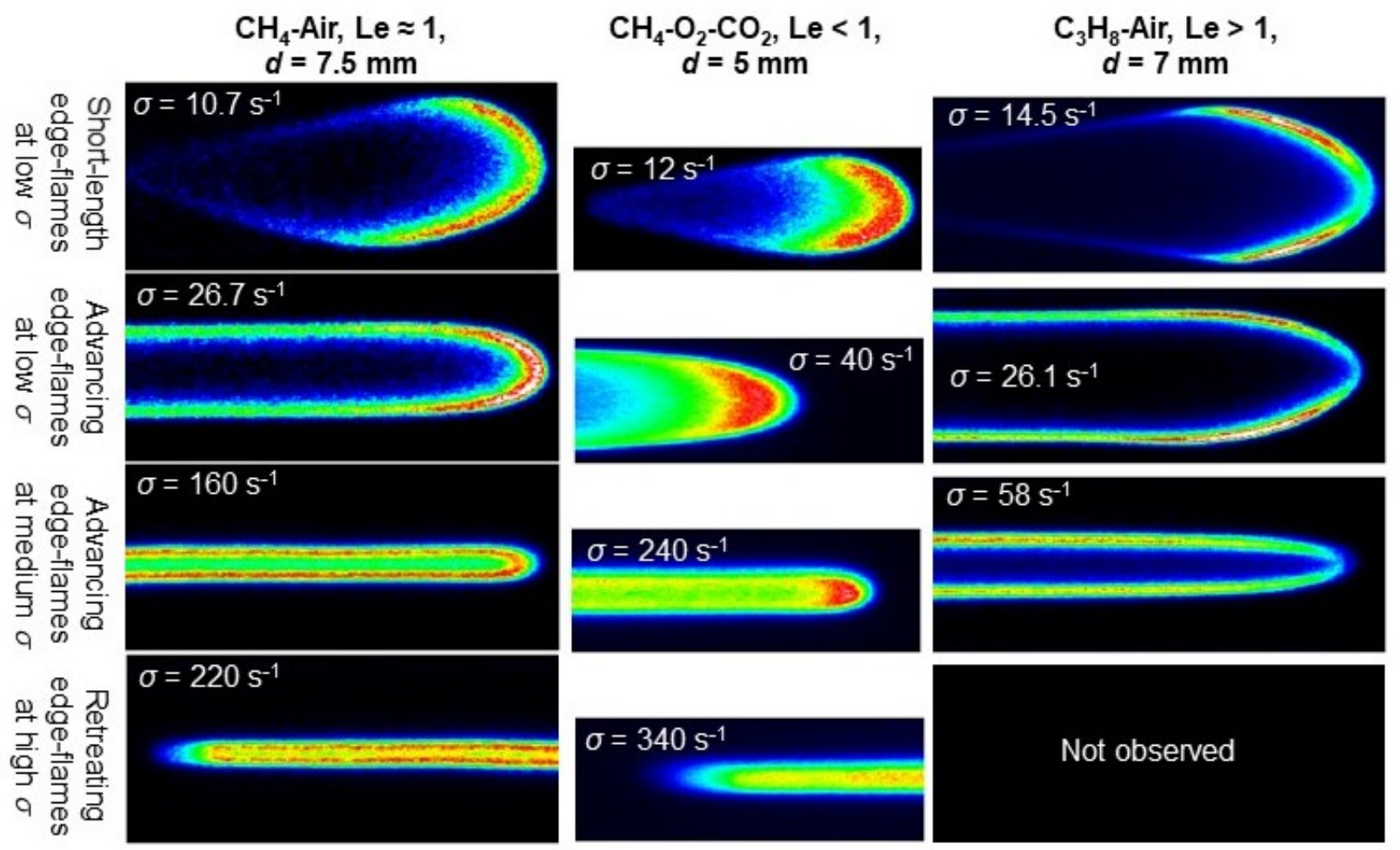

Figure 1. False-color direct images of twin premixed edge-flames. Mixtures for these images are indicated in Table 1. Global strain rate $(\sigma)$ shown in each image. All flames propagate from left to right and the height of each image is scaled with the jet spacing $(d)$.

\section{Results and Discussion}

\subsection{Twin Premixed Edge Flames}

Figure 1 shows images of twin edge-flames for varying $\sigma$ and $L e$. The structures consist of two nearly-flat parallel twin-flames that fold over and connect at the flame edge. The twin-flames trail advancing edges and lead retreating edges. Since gas velocities decrease from the jet exits toward the central stagnation plane, at low $\sigma$ and thus low jet exit velocities $\left(U_{1}, U_{2}\right)$, the twin-flames stabilize near the jet exits where the local convection velocity equals $S_{L}$. As $\sigma$ increases, the twin-flames stabilize closer to the stagnation plane and for high $\sigma$ at low Le the flames completely merge. For $\mathrm{CH}_{4}$-air mixtures $(L e \approx 1)$ the flame edge and trailing flames show nearly equal intensity, which is expected since for $L e \approx 1$, curvature effects on local flame temperature and burning rates are minimal [17]. An exception occurs for very low $\sigma$ where flames are very close to the jet exits and thus subject to substantial heat losses, thereby reducing flame temperature, burning rate and emission intensity. For $\mathrm{C}_{3} \mathrm{H}_{8}$-air mixtures 
$(L e>1)$ leading edges are less intense than the trailing twin-flames indicating weaker reaction due to curvature [12]. For $\mathrm{CH}_{4}-\mathrm{O}_{2}-\mathrm{CO}_{2}$ mixtures $(L e<1)$ curved leading edges are more intense than the trailing twin-flames and there are noticeable bulges toward the jet exits in the curved region, indicating higher local $S_{L}$ than in the trailing twin-flames, consistent with prior experiments on stationary twin edge-flames [12].

Figure 2 shows scaled values of $U_{\text {edge }}$ for twin edge-flames in $\mathrm{CH}_{4}$-air, $\mathrm{C}_{3} \mathrm{H}_{8}$-air and $\mathrm{CH}_{4}-\mathrm{O}_{2}-\mathrm{CO}_{2}$ mixtures for varying scaled global strain rate $\varepsilon$. Figure 2a includes theoretical predictions [7] for $L e=1$ with $\kappa=0$ and $\kappa=0.15$, corresponding approximately to $\geq 5.6 \%$ and $\approx 5.3 \% \mathrm{CH}_{4}$-air, respectively (see Table 1); this theory predicts similar monotonic behavior for all $0 \leq \kappa \leq 0.10$ with transition to nonmonotonic behavior above $\kappa \approx 0.12$. Space limitations preclude showing corresponding plots of unscaled $U_{e d g e}$ vs. $\sigma$, an example is given in Supplemental Data, Figure S2. For some cases high- $\varepsilon$ extinction limits could not be obtained due to experimental limitations. Key features of Figure 2 include:

- All cases show both low- $\varepsilon$ and high- $\varepsilon$ extinction limits with maximum $U_{\text {edge }}$ at intermediate $\varepsilon$.

- Both positive (ignition) and negative (extinction) edge-flame speeds were obtained except for Le $>1$ where negative $U_{\text {edge }}$ could not be obtained (despite considerable effort.) The reason is not clear considering that theory $[7,8]$ predicts negative twin edge-flame speeds at large $\varepsilon$ for all Le, though only for very narrow ranges of $\varepsilon$ at higher $L e$.

- The results are consistent with theory for $L e=1$ [7] in terms of behavior for nearly-adiabatic (low$\kappa)$ and highly nonadiabatic conditions $(\kappa \approx 0.15)$ (these authors did not provide corresponding predictions for Le $\neq 1$, thus, no corresponding predictions are shown in Figs. 2b-c).

- Edge-flames are much stronger at lower Le as evidenced by higher scaled $U_{\text {edge }}$ and larger maximum $\varepsilon$. 
- For each mixture family, the maximum value of the scaled extinction strain rate parameter $\varepsilon$ is nearly the same for every dilution level, indicating that $\varepsilon$ is an appropriate scaling parameter to characterize extinction behavior.

- To obtain $U_{\text {edge }} / S_{L} \approx 1$ at intermediate $\varepsilon$ for the $L e \approx 1$ case $\left(\mathrm{CH}_{4}\right.$-air $)$ as theoretically predicted [7], $U_{\text {edge }} / S_{L}$ was scaled with density ratio $\rho_{u} / \rho_{b}$ rather than $\left(\rho_{u} / \rho_{b}\right)^{1 / 2}$ as with nonpremixed flames (see Introduction). This might be expected because the low-density products are partly trapped between the twin-flames, analogous to expanding spherical flames where mass conservation mass dictates that flame fronts propagate outward at a speed of $\left(\rho_{u} / \rho_{b}\right) S_{L}$ rather than $S_{L}$ itself.

- For all but the high-Le case, at sufficiently low $\varepsilon$ continuous trains of "short-length" edge-flames typically $1 \mathrm{~cm}$ long, originating at the hot-wire and propagating lengthwise from one end of the slot-jet to the other, were observed. Both (positive) leading-edge speed and (negative) trailingtail speed are reported in Figure 2; these are nearly equal, indicating that short-length flames have constant length. 

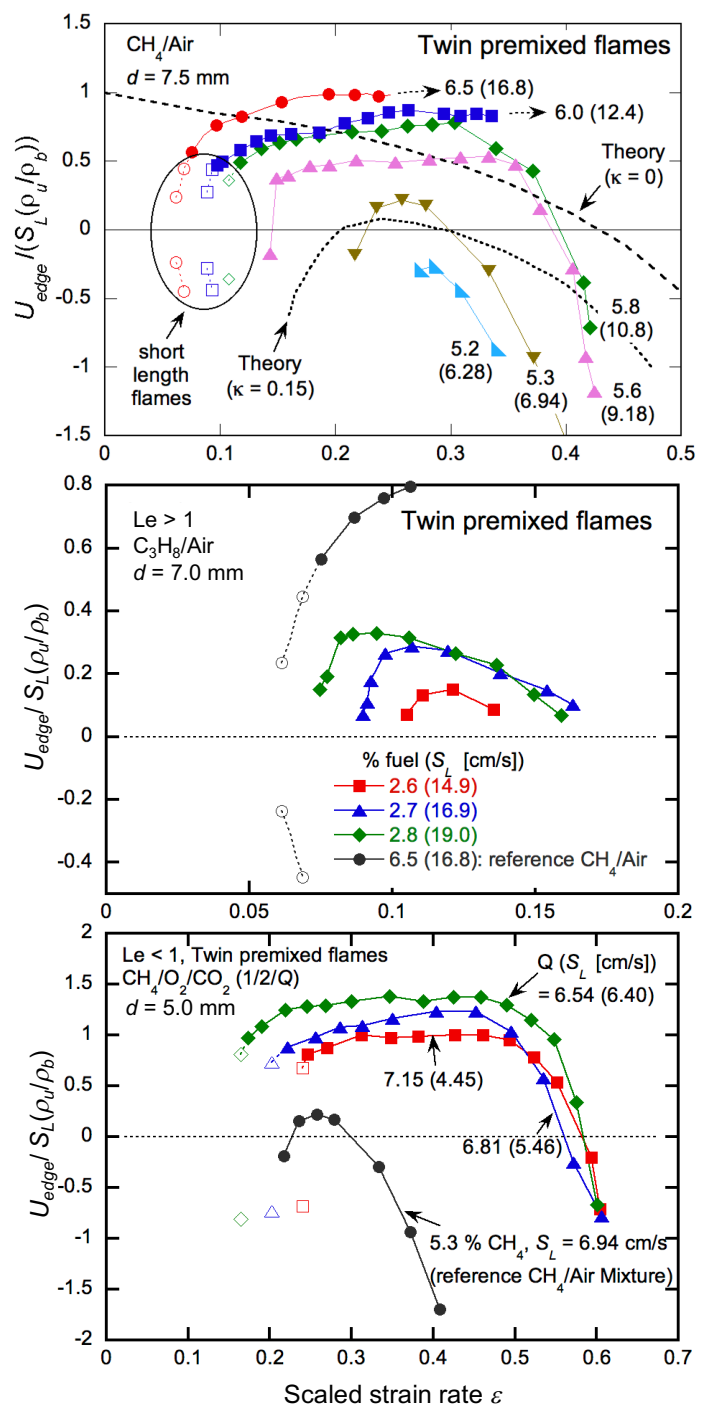

Figure 2. Effect of scaled strain rate $(\varepsilon)$ on scaled edge-flame speed $\left(U_{\text {edge }}\right)$ for twin premixed edgeflames. Top: $\mathrm{CH}_{4} /$ air, $\left.d=7.5 \mathrm{~mm}, L e \approx 1\right)$; middle: $\mathrm{C}_{3} \mathrm{H}_{8} /$ air, $d=7.0 \mathrm{~mm}$; $L e>1$; lower: $\mathrm{CH}_{4} / \mathrm{O}_{2} / \mathrm{CO}_{2}$, $d=5.0 \mathrm{~mm}, L e<1$. For the latter two plots, a comparison $\mathrm{CH}_{4} /$ air $(L e \approx 1)$ case is shown. Number associated with each curve refers to fuel volume percent (upper, middle) or dilution (lower) with corresponding $S_{L}$ in parentheses. Dashed lines and open symbols denote short-length flames (see text); positive speed refers to leading head and negative speed refers to trailing tail. Also shown in part (a) are theoretical predictions [7] for $L e=1, \kappa=0$ and $\kappa=0.15$.

Two possible mechanisms for "short-length" flames are proposed. One is that thermal expansion (not incorporated in theory [7]) locally increases strain rate (thus $\varepsilon$ ) enough in the edge vicinity to shift $U_{\text {edge }}$ from negative to positive values; once the edge passes the trailing twin-flames experience lower strain corresponding to negative $U_{\text {edge }}$ thus leading to a trailing extinction wave. After the extinction wave passes the fresh mixture is re-ignited by the hot-wire and another short-length flame ensues. A 
second possibility is that Daou et al. [7] (their Fig. 9) show a window of conditions for $\varepsilon \approx 0.15-0.20$ and $\kappa \approx 0.10-0.15$ where both positive and negative values of $U_{\text {edge }}$ having nearly equal magnitudes are predicted; Figure 3 shows that this range of $\varepsilon$ and $\kappa$ is close to that where short-length flames are observed experimentally.

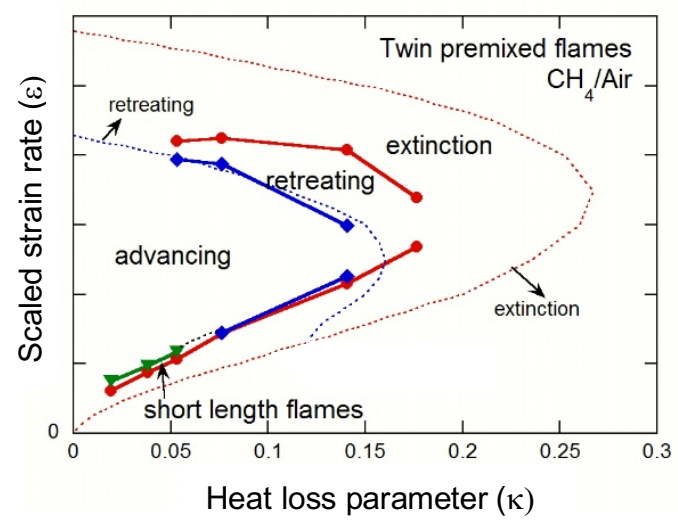

Figure 3. Map in $\boldsymbol{\kappa}-\boldsymbol{\varepsilon}$ space of propagation modes and extinction limits for twin premixed edge-flames. Solid curves: experimental results for $\mathrm{CH}_{4} /$ air mixtures; dashed curves: theoretical predictions [7] for $L e=1$.

Figure 3 shows maps of flame behavior in $\kappa-\varepsilon$ space for experiments on twin premixed edge-flames in $\mathrm{CH}_{4}$-air mixtures $(L e \approx 1)$ along with theoretical predictions for $L e=1$ [7] (these authors did not provide corresponding maps for $L e \neq 1$ ). Similarities between the two plots include (1) the low- $\varepsilon$ boundary between advancing and retreating edge-flames occurs at $\varepsilon \approx 1.5 \kappa,(2)$ the corresponding high- $\varepsilon$ boundary occurs near $\varepsilon=0.4$ and is less affected by $\kappa$ (because it caused primarily by insufficient flame residence time, not heat losses) and (3) the two limits converge at $\varepsilon \approx 0.25, \kappa \approx 0.15$. The theory, however, predicts much larger ranges of conditions resulting in negative $U_{\text {edge }}$ than were observed experimentally. We speculate that this may because the predictions assume volumetric (radiative) loss, not gradient-driven conductive loss to the jet exits as in the experiments. 


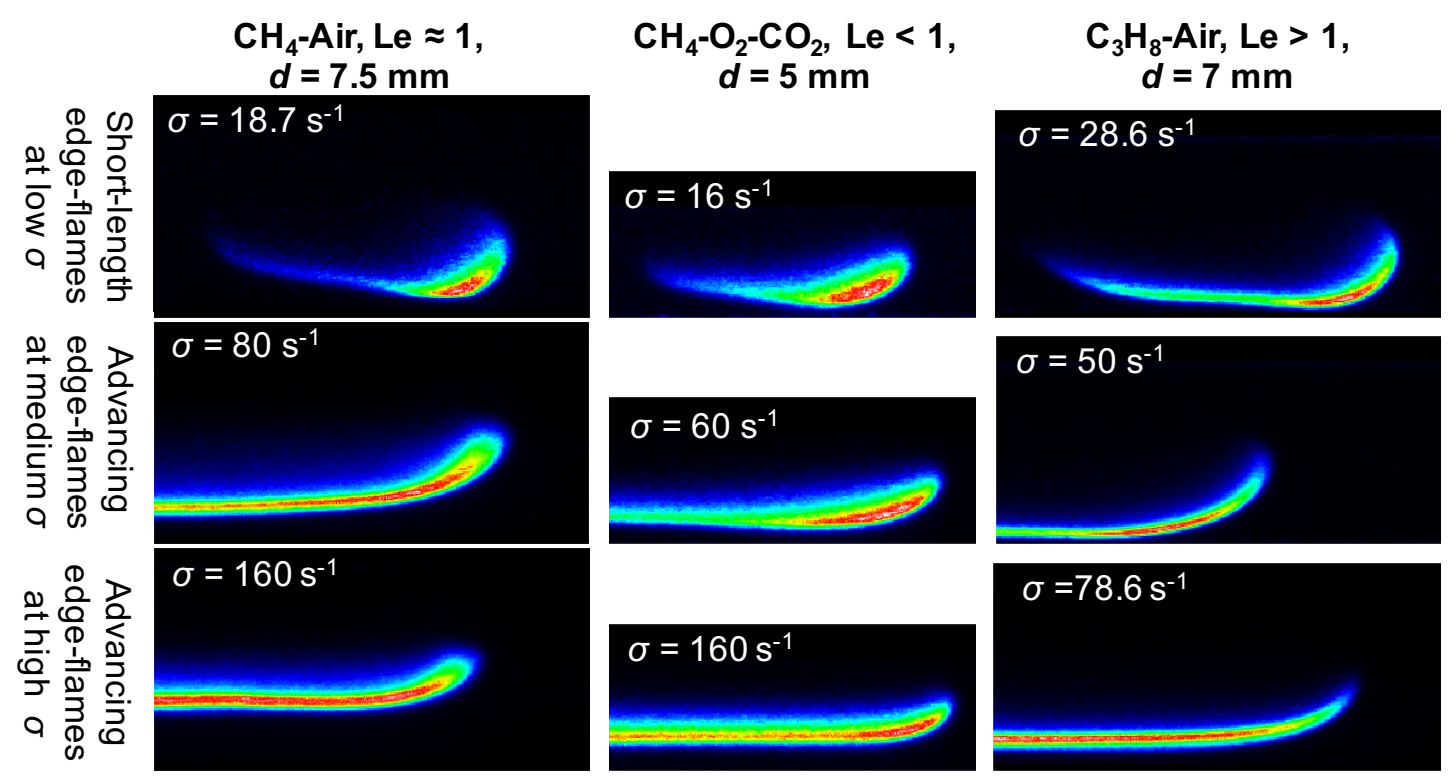

Figure 4. Direct images of single premixed edge-flames. Mixtures for these images are indicated in Table 1. Global strain rate $(\sigma)$ shown in each image. Reactive mixture flows from bottom, cold inert from top. All flames propagate from left to right and the height of each image is scaled with the jet spacing $(d)$.

\subsection{Single Premixed Edge Flames}

Figure 4 shows images of single edge-flames for varying $\sigma$ and Le. Images are shown only for reactive/inert flow from the bottom/top jet, though results were similar with the flows reversed, indicating that buoyancy effects (discussed at length in [9]) were insignificant. The structures consist of distinct edges with single quasi-planar premixed flames trailing advancing edges and leading retreating edges. Since all single flames have distinct ends with decreasing intensity near the end, unlike twinflames (Figure 1) there is no obvious visual effect of Le on edge-flame burning intensity. The flame sheet near the tip suffers massive heat losses which decreases its propagation speed, thus it retreats (tilting upward) toward the stagnation plane where the convection velocity is lower. If the jets reversed (reactive on top, inert on the bottom) the tilting is downward. Figure 5 shows scaled values of $U_{\text {edge }}$ for single edge-flames in $\mathrm{CH}_{4}$-air, $\mathrm{C}_{3} \mathrm{H}_{8}$-air and $\mathrm{CH}_{4}-\mathrm{O}_{2}-\mathrm{CO}_{2}$ mixtures for varying scaled global strain rate $\varepsilon$. As with twin edge-flames, all cases show both low- $\varepsilon$ and high- $\varepsilon$ extinction limits with maximum 
$U_{\text {edge }}$ at intermediate $\varepsilon$, with lower $L e$ exhibiting higher scaled $U_{\text {edge }}$ and larger maximum $\varepsilon$. There are several key differences from twin edge-flames, however:

- Much stronger mixtures (higher $S_{L}$ ) are required to avoid extinction and the maximum values of $\varepsilon$ for which flames exist are far lower. This is somewhat expected because of the heat loss caused by the cold inert gas opposing the reactive mixture compared to the mutual support provided by the back-to-back configuration of twin-flames. The single premixed flame is somewhat analogous to nonpremixed flames where heat-loss occurs from both sides of a single reaction sheet. Following this analogy, it might be more appropriate to scale $\varepsilon$ for single edgeflames as $\beta\left(\sigma \alpha / 2 S_{L}^{2}\right)^{1 / 2}$ rather than $\left(\sigma \alpha / 2 S_{L}^{2}\right)^{1 / 2}$, in which case the maximum values of $\varepsilon$ would be similar for the single- and twin-flame cases; nevertheless, to facilitate comparisons we chose to employ $\left(\sigma \alpha / 2 S_{L}^{2}\right)^{1 / 2}$ scaling for both premixed cases.

- No retreating edge-flames were obtained despite considerable experimental effort; this is consistent with theory [18] which predicts negative $U_{\text {edge }}$ values exist for twin but not for single premixed flames.

- To obtain $U_{e d g e} / S_{L} \approx 1$ at intermediate $\varepsilon$ for the $L e \approx 1$ case $\left(\mathrm{CH}_{4}\right.$-air) as theoretically predicted, $U_{\text {edge }} / S_{L}$ was scaled with $\left(\rho_{u} / \rho_{b}\right)^{1 / 2}$ as with nonpremixed flames (see Introduction). This might be expected because the thermal expansion induced flow-field resulting from the single-flame with a single reactive sheet more closely resembles nonpremixed flame than twin premixed flames.

- "Short-length" edge-flames were observed at low $\varepsilon$ for all mixtures tested. This is somewhat surprising considering that theory does not predict negative edge speeds for single premixed flames and thus a trailing extinction wave required for short-length flames would not be expected. 
Figure 6 shows maps of behavior of single premixed $\mathrm{CH}_{4}$-air edge-flames in $\kappa-\varepsilon$ space $(L e \approx 1)$ (unlike twin-flames, no corresponding theoretical map for single-flames has been reported that could be compared to experiments.) The range of both $\kappa$ and $\varepsilon$ where flames could be sustained is an order of magnitude lower than for twin-flames, but, as with twin-flames, the high- $\varepsilon$ limit is much less affected by heat loss $\kappa$ than the low- $\varepsilon$ limit. Unlike twin-flames, short-length flames were observed for all $\kappa$, however, these values of $\kappa$ are all within the range where short-length flames were observed for twin edge-flames.

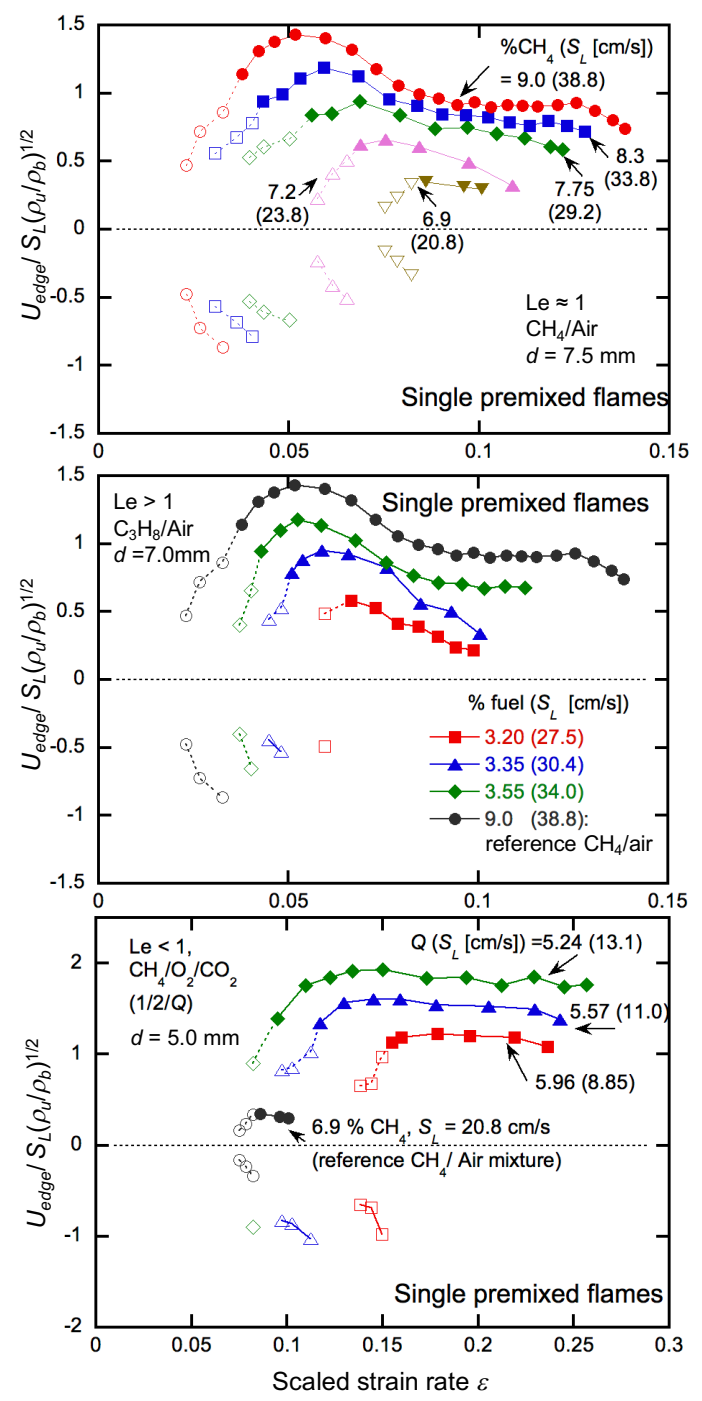

Figure 5. Effect of scaled strain rate $(\varepsilon)$ on scaled edge-flame speed ( $\left.U_{\text {edge }}\right)$ for single premixed edgeflames. Upper: $\mathrm{CH}_{4} /$ air, $\left.d=7.5 \mathrm{~mm}, L e \approx 1\right)$; middle: $\mathrm{C}_{3} \mathrm{H}_{8} /$ air, $d=7.0 \mathrm{~mm} ; L e>1$; lower: $\mathrm{CH}_{4} / \mathrm{O}_{2} / \mathrm{CO}_{2}$, $d=5.0 \mathrm{~mm}, L e<1$. For the latter two plots, a comparison $\mathrm{CH}_{4} /$ air $(L e \approx 1)$ case is shown. Number associated with each curve refers to fuel volume percent (upper, middle) or dilution (lower) with 
corresponding $S_{L}$ in the parentheses. Dashed lines and open symbols correspond to short-length flames (see text); positive speed refers to leading head and negative speed refers to trailing tail.

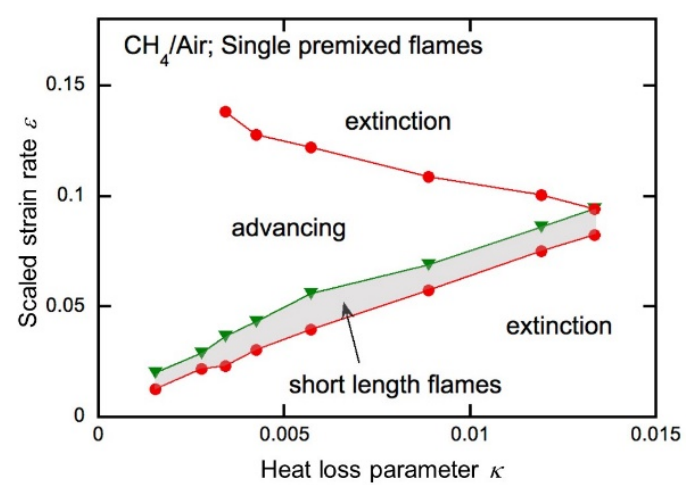

Figure 6. Map in $\kappa-\varepsilon$ space of propagation modes and extinction limits for single premixed edge-flames for experiments in $\mathrm{CH}_{4}$ /air mixtures.

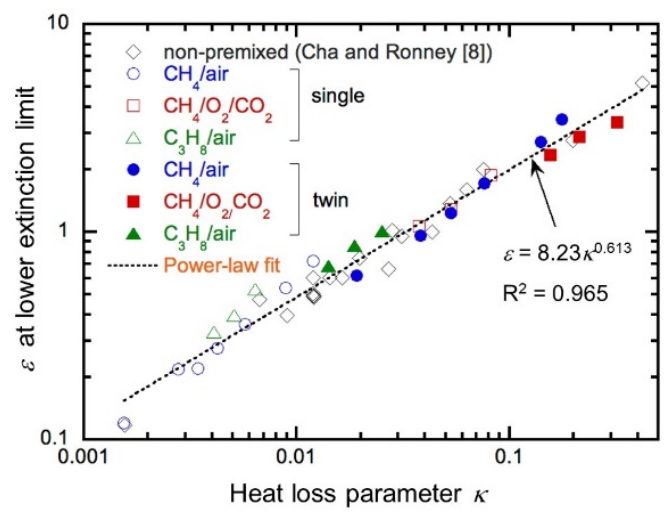

Figure 7. Correlation of scaled strain rate $\varepsilon$ (scaled with $\beta$ as for non-premixed flames [9]) at the lowstrain (heat-loss-induced) extinction limit with heat loss parameter $(\kappa)$ for all conditions tested, along with corresponding data for non-premixed flames [9].

\subsection{Low-strain extinction limit correlation}

While the mechanism of the high- $\varepsilon$ extinction limit for strained flames is well-established and is mostly unaffected by the heat loss parameter $\kappa$, the low- $\varepsilon$ limit is strongly affected by $\kappa$. Figure 7 shows the correlation between the $\kappa$ and $\varepsilon$ (scaled as $\beta\left(\sigma \alpha / 2 S_{L}^{2}\right)^{1 / 2}$ for comparison with nonpremixed flames [9]) at the low- $\varepsilon$ extinction limit. Twin premixed, single premixed and nonpremixed flames all follow a simple power-law correlation over more than two decades of $\kappa$, which is close to $\varepsilon \sim \kappa^{1 / 2}$ or (in dimensional terms) $\sigma \sim \alpha / d^{2}$. For nonpremixed flames this correlation is equivalent to equating the 
mixing layer thickness $\delta \sim(\alpha / \sigma)^{1 / 2}$ to the jet spacing $d$, which is clearly the condition for heat losses to dominate. For premixed flames, this correlation is equivalent to the typical quenching criterion [19] of constant Peclet number $=S_{L} d / \alpha$ with the flame stabilized near the jet exits and thus $S_{L} \sim U \sim \sigma d$. Consequently, the $\varepsilon \sim \kappa^{1 / 2}$ scaling is logical, though it is perhaps surprising that all three flame types follow essentially the same correlation.

\section{Conclusions}

An experimental study of twin and single premixed edge-flames revealed rich varieties of structures depending on configuration (single or twin), mixture strength, Lewis number, strain rate and density ratio. Most phenomena (flame shapes, burning intensities, occurrence of propagating and retreating edge-flames, propagation speeds relative to $S_{L}$, high- $\varepsilon$ and low- $\varepsilon$ extinction limits) could be interpreted based on theoretical developments via scaled parameters characterizing strain rate $(\varepsilon)$ and heat loss $(\kappa)$. The two experimental observations not predicted by theory were (1) the absence of retreating edges for high-Le $\left(\mathrm{C}_{3} \mathrm{H}_{8}\right.$-air) twin edge-flames and (2) continuous trains of "short-length" edge-flames at very low $\varepsilon$. While no reason could be identified for the former, we hypothesize that the latter may be due to thermal expansion effects not included in the aforementioned theories. Direct Numerical Simulation (DNS) of edge-flames in the counterflow apparatus with heated flame anchors would be required to test this hypothesis. Other DNS studies [20] modeling preheated reactants having appreciable low-temperature chemistry have identified far more varied edge-flame structures than those observed here; experiments to test these predictions would be relevant to autoignition in diesel engine combustion.

\section{Acknowledgements}


This work was supported by the U. S. National Science Foundation under grant CBET-1236892.

MSC was supported by King Abdullah University of Science and Technology.

\section{References}

[1] S. Karami, E.R. Hawkes, M. Talei, J.H. Chen, Combust. Flame 169 (2016) 110-128.

[2] J.D. Buckmaster, Prog. Energy Combust. Sci. 28 (2002) 435-475.

[3] S.H. Chung, Proc. Combust. Inst. 31 (2007) 877-892.

[4] T.C. Lieuwen, Unsteady Combustor Physics, Cambridge University Press, 2012.

[5] R. Knauss, C. Pantano, J. Comp. Phys. 296 (2015) 209-240.

[6] R. Daou, J. Daou, J. Dold, Proc. Combust. Inst. 29 (2002) 1559-1564.

[7] R. Daou, J. Daou, J. Dold, Combust. Theory and Modelling 7 (2003): 221-242.

[8] J. Daou, A. Linan, Combust. Flame 118 (1999) 479-488

[9] M.S. Cha, P.D. Ronney, Combust. Flame 146 (2006) 312-328.

[10] H. Song, P. Wang, R.S. Boles, D. Matinyan, H. Prahanphap, J. Piotrowicz, P.D. Ronney, Proc. Combust. Inst. 36 (2007) 1403-1409.

[11] G.R. Ruetsch, L. Vervisch, A. Liñán, Phys. Fluids 7 (1995) 1447-1454.

[12] J.B. Liu, P.D. Ronney, Combust Sci. Tech. 144 (1999) 21-46.

[13] K. Takita, S. Sakaguchi, G. Masuya, Combust. Flame 132 (2003) 343-351

[14] K. Takita, M. Sado, G. Masuya, S. Sakaguchi, , Combust. Flame 136 (2004) 364-370

[15] K. Seshadri, F.A. Williams, Int. J. Heat Mass Trans. 21 (1978) 251-253.

[16] Z. Qin, V. Lissianski, H. Yang, W. C. Gardiner, Jr., S. G. Davis, H. Wang, Proc. Combust. Inst. 28 (2000) 1663-1669.

[17] F.A. Williams, Combustion Theory, $2^{\text {nd }}$ Ed., Benjamin-Cummins, 1985.

[18] T.G. Vedarajan, J.D. Buckmaster, P.D. Ronney, P. D., Proc. Combust. Inst. 27 (1998) 537-544.

[19] J. Jarosinski, Combust. Flame 50 (1983) 167-175.

[20] A. Krisman, E.R. Hawkes, M. Talei, A. Bhagatwala, J.H. Chen, Proc. Combust. Inst. 35 (2015) 1999-2006. 


\section{Supplemental Data}

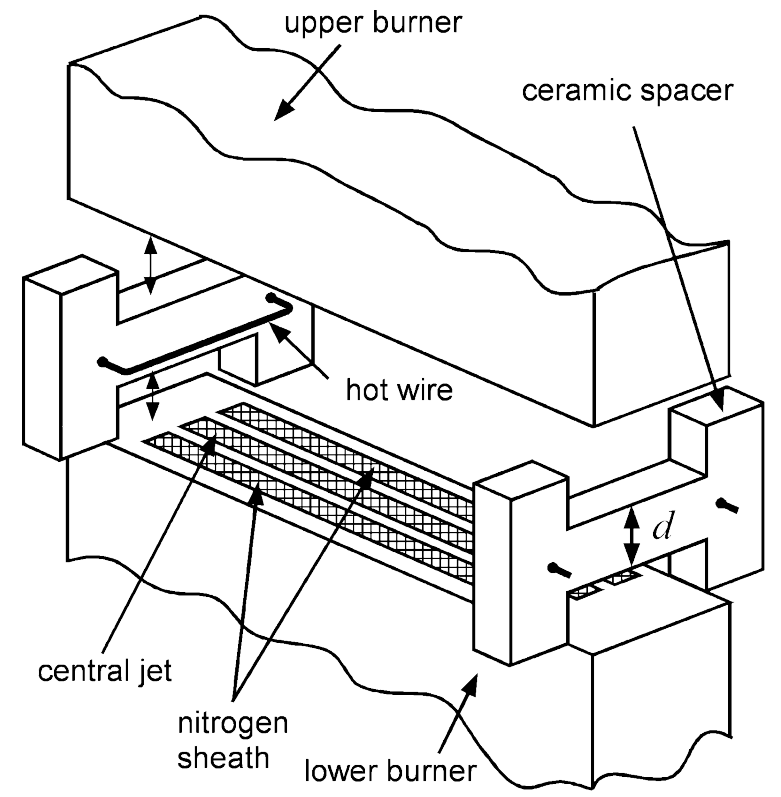

Figure S1. Schematic of the experimental apparatus $[9,10]$.

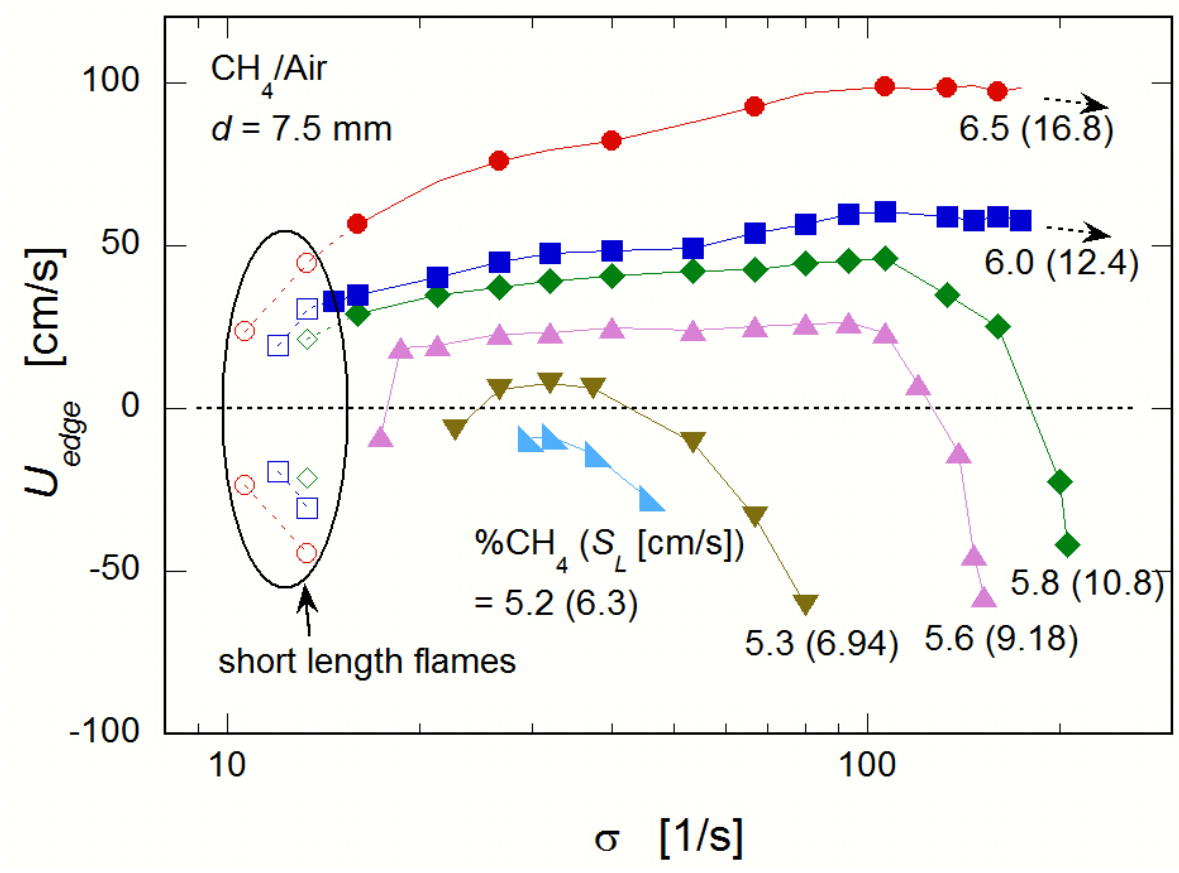

Figure S2. Effect of strain rate $(\sigma)$ on edge-flame speed $\left(U_{\text {edge }}\right)$ for twin premixed edge-flames in $\mathrm{CH}_{4} /$ air mixtures, $d=7.5 \mathrm{~mm}, \mathrm{Le} \approx 1$. This plot corresponds to the raw data used for the scaled values shown in Figure 2 (upper). Number associated with each curve refers to fuel volume percent (upper, middle) or dilution (lower) with corresponding $S_{L}$ in parentheses. Dashed lines and open symbols denote short-length flames (see text); positive speed refers to leading head and negative speed refers to trailing tail. 